\title{
Impact of mitochondrial alternative oxidase expression on the response of Nicotiana tabacum to cold temperature
}

\author{
Jia Wang ${ }^{a, b}$, Nirusan Rajakulendran ${ }^{a}$, Sasan Amirsadeghia and Greg C. Vanlerberghe ${ }^{a, b, *}$ \\ aDepartment of Biological Sciences, University of Toronto Scarborough, 1265 Military Trail, Toronto, ON M1C 1A4, Canada \\ ${ }^{b}$ Department of Cell and Systems Biology, University of Toronto Scarborough, 1265 Military Trail, Toronto, ON M1C 1A4, Canada
}

\author{
Correspondence \\ *Corresponding author, \\ e-mail: gregv@utsc.utoronto.ca \\ Received 5 November 2010; \\ revised 4 February 2011
}

doi:10.1111/j.1399-3054.2011.01471.x
The plant mitochondrial electron transport chain (ETC) includes a non-energy conserving alternative oxidase (AOX) thought to dampen reactive oxygen species (ROS) generation by the ETC and/or facilitate carbon metabolism by uncoupling it from ATP turnover. When wild-type (WT) Nicotiana tabacum grown at $28^{\circ} \mathrm{C} / 22^{\circ} \mathrm{C}$ (light/dark) were transferred to $12^{\circ} \mathrm{C} / 5^{\circ} \mathrm{C}$, they showed a large induction of leaf Aox1a mRNA and AOX protein within $24 \mathrm{~h}$. Transfer to cold also resulted in a large accumulation of monosaccharides, an increase in transcript level of genes encoding important ROS-scavenging enzymes and a moderate increase in lipid peroxidation. Transgenic plants with suppressed AOX level showed less cold-induced sugar accumulation than WT while transgenic plants with enhanced AOX levels showed enhanced sugar accumulation. This is inconsistent with the hypothesis that AOX acts to burn excess carbohydrate, but rather suggests a role for AOX to aid sugar accumulation, at least during cold stress. At $28^{\circ} \mathrm{C} / 22^{\circ} \mathrm{C}$, plants with suppressed AOX had elevated levels of lipid peroxidation compared with WT, while plants with enhanced AOX had reduced lipid peroxidation. This is consistent with the hypothesis that AOX dampens ROS generation and oxidative damage. However, this inverse relationship between AOX level and lipid peroxidation did not hold upon shift to cold. Under this stress condition, plants with strong suppression of AOX show enhanced induction of ROS-scavenging enzymes compared with WT and decline in lipid peroxidation. These data suggest that, under stress conditions, the lack of AOX enhances a mitochondrial stress-signaling pathway able to increase the ROS-scavenging capacity of the cell.

\section{Introduction}

Besides cytochrome oxidase, all plants have an additional terminal respiratory oxidase called alternative oxidase (AOX) that catalyzes the oxidation of ubiquinol and reduction of $\mathrm{O}_{2}$ to $\mathrm{H}_{2} \mathrm{O}$ (Finnegan et al. 2004). AOX is non-proton pumping and as it bypasses proton-pumping complexes III and IV, electron flow to AOX dramatically reduces the energy yield of respiration. AOX is encoded by a small nuclear gene family and is an interfacial membrane protein on the matrix side of the inner mitochondrial membrane, existing as a homodimer. Many AOX gene family members contain a conserved regulatory Cys residue that confers tight biochemical control over the AOX enzyme and links its activity to the prevailing metabolic conditions within the mitochondrion.

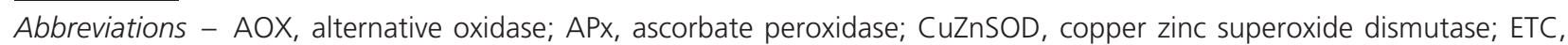
electron transport chain; FeSOD, iron superoxide dismutase; Fru, fructose; Glu, glucose; GPx, glutathione peroxidase; MDA, malondialdehyde; PPFD, photosynthetic photon flux density; ROS, reactive oxygen species; WT, wild-type. 
Because of its non-energy conserving nature, there has been considerable interest to understand the metabolic and physiological role(s) of AOX respiration, besides its well-established role to generate heat in highly specialized thermogenic tissues such as the floral inflorescence of Philodendron bipinnatifidum (Miller et al. 2010). As AOX respiration reduces the otherwise tight coupling among carbon metabolism, electron transport and ATP turnover, it could play a number of roles in the optimization of respiratory metabolism as well as the integration of respiratory metabolism with other metabolic pathways that impact the supply of or demand for carbon skeletons, reducing power and ATP (Finnegan et al. 2004, Vanlerberghe et al. 2009). Another central hypothesis is that $A O X$ respiration may act to dampen reactive oxygen species (ROS) generation by the mitochondrial electron transport chain (ETC), as its energy-dissipating nature will act to moderate membrane potential (Purvis and Shewfelt 1993). However, as recently summarized, reverse genetic experiments have yielded contrasting results regarding levels of ROS, levels of oxidative damage and status of the ROS-scavenging network in plants lacking AOX (Vanlerberghe et al. 2009).

A major effort is needed to identify the developmental, metabolic, physiological and environmental conditions under which non-energy conserving respiration may be of benefit to plants. Interestingly, many studies indicate that the expression of distinct AOX gene family members is strongly induced by numerous biotic and abiotic stresses (Finnegan et al. 2004, Simons and Lambers 1999, Vanlerberghe et al. 2009). For example, studies in many plant species have shown a sharp increase in AOX transcript and/or protein after transfer to or growth at low temperature (Armstrong et al. 2008, Campbell et al. 2007, Fiorani et al. 2005, Gonzàlez-Meler et al. 1999, Ito et al. 1997, Mizuno et al. 2008, Sugie et al. 2006, Taylor et al. 2002, Usadel et al. 2008, Umbach et al. 2009, Vanlerberghe and McIntosh 1992, Watanabe et al. 2008). Also, a number of studies have used an oxygen isotope discrimination technique to determine the steady-state flux of electrons to AOX in vivo (i.e. AOX engagement). Such changes in AOX engagement have been assessed in response to instantaneous (i.e. minutes to hours), short-term (several hours to several days) and long-term (weeks to months) changes in temperature. For example, MacFarlane et al. (2009) found that instantaneous changes in temperature had little impact on the relative partitioning of electrons between AOX and the cytochrome pathway in three plant species over the temperature range of $17-36^{\circ} \mathrm{C}$. Similarly, Guy and Vanlerberghe (2005) found relatively little instantaneous changes in AOX engagement over a wide range of measurement temperatures in tobacco, even in plants overexpressing AOX protein. However, in chilling-sensitive maize, a short-term cold treatment ( 5 days at $5^{\circ} \mathrm{C}$ ) was associated with a dramatic decline in cytochrome pathway activity and increase in AOX engagement, such that approximately $60 \%$ of total respiration was occurring via AOX (Ribas-Carbo et al. 2000). Similarly, Armstrong et al. (2008) found a transient increase in AOX engagement in Arabidopsis with sustained chilling (over first 10 days at $5^{\circ} \mathrm{C}$ ), followed by a return to lower engagement in the longer term. In this case, the transient increase in AOX engagement occurred even without any corresponding increase in AOX protein. Alternatively, Fiorani et al. (2005) found that Arabidopsis plants grown at $12^{\circ} \mathrm{C}$ had higher AOX protein than warm-grown plants and that growth at the low temperature was compromised in plants lacking AOX, suggesting an important longer term role for the pathway at low temperature. Also, Searle et al. (2010) found that field-grown alpine grasses had higher AOX protein (relative to cytochrome pathway) in the cold months and other studies have also found higher AOX protein in plants developed in the cold compared with warm-grown plants (Campbell et al. 2007, Umbach et al. 2009). On the basis of results to date, it seems possible that the response of AOX to temperature will be species- and tissue-dependent, dependent on the severity and length of the cold treatment and dependent on the developmental or physiological status of the plant at the time of cold treatment. Nonetheless, results to date clearly show that AOX respiration commonly becomes more prevalent during both sustained short-term chilling and longer term growth at low temperature.

The functional importance of AOX under low temperature conditions remains largely unknown. For example, its activity might satisfy particular metabolic needs or, alternatively, its activity might be part of a coordinated response of the ROS network needed to balance ROS generation and scavenging. Like many stresses, low temperature is known to alter this network and the ROS themselves might be acting as damaging toxic molecules and/or useful signaling molecules (Suzuki and Mittler 2006). In this study, we have used transgenic plants with modified levels of a stress-inducible leaf AOX to investigate the physiological role(s) of this pathway after short-term transfer of tobacco to a lower growth temperature.

\section{Materials and methods}

\section{Plant material and growth conditions}

Tobacco (Nicotiana tabacum cv Petit Havana SR1) was used for all experiments. Transgenic plant lines with 
suppressed levels of AOX protein (RI29, RI9) because of the presence of an Aox1a RNA interference construct or elevated levels of AOX protein (B7, B8) because of the presence of an Aox1a transgene driven by a constitutive promoter have been previously described (Amirsadeghi et al. 2006, Vanlerberghe et al. 1998). Seeds of wildtype (WT) and transgenic plants were germinated in vermiculite for 3 weeks and then transferred to 4 -in. plastic pots containing a general purpose growing medium consisting of four parts Pro-mix BX (Premier Horticulture Ltd, Rivière, Rivière-du-Loup, Quebec, Canada) and one part vermiculite (Therm-O-Rock East Inc., New Eagle, PA). Plants were raised in controlled-environment growth chambers (Model PGR-15; Conviron, Winnipeg, Canada) with a 16 -h photoperiod, a temperature of $28^{\circ} \mathrm{C} / 22^{\circ} \mathrm{C}$ (light/dark), a photosynthetic photon flux density (PPFD) of $120 \mu \mathrm{mol} \mathrm{m}^{-2} \mathrm{~s}^{-1}$ (unless indicated otherwise) and a relative humidity of $60 \%$. Plants were irrigated daily with a $10 \times$ diluted Hoagland's solution (Hoagland and Arnon 1950). Three weeks after transfer to pots, fully developed leaves (leaves 4 and 5) of one series of plants were sampled for various measures $(0 \mathrm{~h})$. Parallel series of plants were then transferred to a lower growth temperature $\left[12^{\circ} \mathrm{C} / 5^{\circ} \mathrm{C}\right.$ (light/dark)] and sampled at 24,48 or $72 \mathrm{~h}$ after transfer. The transfer and all sampling were done at $12 \mathrm{~h}$ into the light period.

\section{Transcript analyses}

RNA was extracted from leaf tissues according to the method described by Vanessa et al. (2008) in order to overcome the interference caused by polysaccharide accumulation after transfer of plants to low temperature. Northern blot analyses were performed following the method described by Sieger et al. (2005). To generate the probes for hybridization, partial sequences for each designated gene were amplified with an RT-PCR kit (Access RT-PCR; Promega, Madison, WI) from tobacco leaf RNA and cloned into pGEM-T Easy Vector (Promega). The cDNA fragments were then excised from these plasmids and used for radioactive labeling. The primer sequences used for cDNA synthesis of ascorbate peroxidase $(\mathrm{APx})$, glutathione peroxidase $(\mathrm{GPx})$, copper zinc superoxide dismutase (CuZnSOD) and iron superoxide dismutase (FeSOD) were as described by Amirsadeghi et al. (2006). For AOX, a full-length tobacco Aox1a cDNA (Vanlerberghe and McIntosh 1994) was used as probe for hybridization. After X-ray film development, Northern blots were quantified by densitometry using an imaging system (Alpha Innotech Corporation, San Leandro, CA) and associated software (AlphaEAseFC).

\section{Protein analyses}

Isolation of mitochondria from leaves (starting with $45 \mathrm{~g}$ of leaf tissue from four individual plants) was done as previously described (Vanlerberghe et al. 1995). The mitochondrial protein was then used for immuno-blot analysis according to the method described by Robson and Vanlerberghe (2002).

\section{Hexose, sucrose and starch analyses}

For hexose, sucrose and starch analyses, the methods of Stitt et al. (1989) and Jones (1981) were followed, with some modification. Leaf tissues were quickly frozen in liquid $\mathrm{N}_{2}$, ground with a mortar and pestle, and then freeze-dried $\left(-50^{\circ} \mathrm{C}, 5 \mathrm{~h}\right)$. Freeze-dried tissue $(6 \mathrm{mg})$ was then extracted three times with $80 \%(\mathrm{v} / \mathrm{v})$ ethanol for $20 \mathrm{~min}$ at $80^{\circ} \mathrm{C}$. The pooled extracts were then treated with $5 \mathrm{mg}$ of activated charcoal, dried in a rotary vacuum system at $40^{\circ} \mathrm{C}$ for $2 \mathrm{~h}$ and dissolved in $1 \mathrm{ml}$ of distilled $\mathrm{H}_{2} \mathrm{O}$ for the analysis of glucose (Glu), fructose (Fru) and sucrose. For starch analysis, the insoluble pellets from the ethanol extraction were solubilized by heating at $95^{\circ} \mathrm{C}$ in $0.1 \mathrm{M} \mathrm{NaOH}$ for $1 \mathrm{~h}$. Following acidification to $\mathrm{pH} 4.9$ with $1 \mathrm{M}$ acetic acid, the suspension was digested overnight at $55^{\circ} \mathrm{C}$ in $1 \mathrm{ml}$ enzyme solution [0.2 M sodium acetate, $\mathrm{pH}$ 5.0, $2 \mathrm{U} \mathrm{ml}^{-1}$ amyloglucosidase (Sigma-Aldrich Canada, Oakville, ON, Canada, A7420), $10 \cup \mathrm{ml}^{-1} \alpha$-amylase (Sigma-Aldrich Canada, A3403)]. Samples were then centrifuged (16 $000 \mathrm{~g}$, $5 \mathrm{~min}$ ) and the Glu level of the supernatant used to assess starch content. The level of Glu, Fru and sucrose in the extracts was measured by an enzymatic assay (Stitt et al. 1989), using a Hewlett Packard 8453 diode array spectrophotometer (Agilent Technologies Canada, Mississauga, ON, Canada).

\section{Lipid peroxidation}

The extent of lipid peroxidation in leaf was estimated by malondialdehyde (MDA) content, as determined by the thiobarbituric acid-reactive-substances assay previously described (Hara et al. 2003), but with some modifications. Six leaf discs $(1.5 \mathrm{~cm}$ in diameter) were quickly sampled, weighed and then homogenized in $4 \mathrm{ml}$ of cold $5 \mathrm{mM}$ potassium phosphate buffer $(\mathrm{pH} 7.0)$ in a pre-cooled mortar and pestle, followed by centrifugation $\left(1000 \mathrm{~g}, 10 \mathrm{~min}, 4^{\circ} \mathrm{C}\right)$. Supernatant $(0.9 \mathrm{ml})$ was then added to a mixture containing $0.1 \mathrm{ml}$ of $2.7 \%$ $(\mathrm{w} / \mathrm{v})$ sodium dodecyl sulfate, $0.25 \mathrm{ml}$ of $20 \%$ acetic acid and $0.25 \mathrm{ml}$ of thiobarbituric acid. A blank sample substituted the supernatant with $0.9 \mathrm{ml}$ potassium phosphate buffer. The samples were then incubated at $98^{\circ} \mathrm{C}$ for $1 \mathrm{~h}$, centrifuged (12000 $\mathrm{g}, 5 \mathrm{~min}$ ) and cooled 
to room temperature. MDA content of the sample was then determined using a spectrophotometric assay (Hara et al. 2003).

\section{Glutathione and ascorbic acid analyses}

Leaf glutathione and ascorbic acid were extracted and measured by a method described by Queval and Noctor (2007). The assays were performed on a high performance multi-detection plate reader (FLUOstar OPTIMA; BMG Labtech, Cary, NC).

\section{Results}

\section{Cold results in a large induction of $\mathrm{AOX}$ in tobacco leaf}

In tobacco and Arabidopsis, Aox1a is the AOX gene family member whose expression has been shown to be highly responsive to growth conditions and stress (Clifton et al. 2005, 2006, Vanlerberghe et al. 2009). WT tobacco maintained a very low level of leaf Aox1a transcript at $28^{\circ} \mathrm{C} / 22^{\circ} \mathrm{C}$ (light/dark), but this level was dramatically higher at 24,48 or $72 \mathrm{~h}$ after transfer to $12^{\circ} \mathrm{C} / 5^{\circ} \mathrm{C}$ (Fig. 1A). The increase in Aox1a transcript
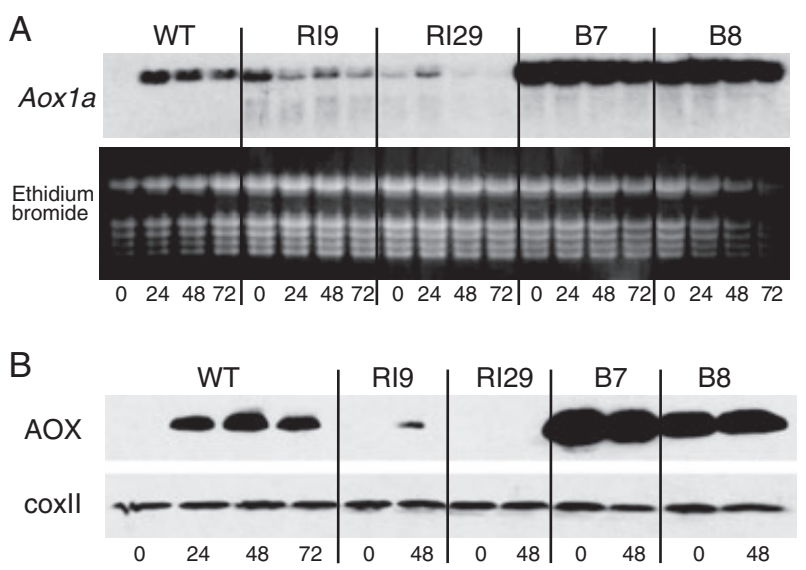

Fig. 1. Levels of Aox1a mRNA and AOX protein in the leaf of WT and transgenic tobacco at different times after transfer of plants from a growth temperature of $28^{\circ} \mathrm{C} / 22^{\circ} \mathrm{C}$ (light/dark) to a growth temperature of $12^{\circ} \mathrm{C} / 5^{\circ} \mathrm{C}$. (A) Northern analysis of Aox1a mRNA level. Note the large increase in Aox1a mRNA level in WT within $24 \mathrm{~h}$ of transfer to cold. Quality of RNA and confirmation of equal loading between lanes was routinely checked by ethidium bromide staining, as shown. (B) Western analysis of the level of AOX protein in isolated mitochondria. Note the large increase in AOX protein in WT plants within $24 \mathrm{~h}$ of transfer to cold. Confirmation of sample quality and equal loading between lanes was routinely checked by also examining levels of cytochrome oxidase subunit II (coxII) protein in the isolated mitochondria, as shown. Mitochondrial proteins $(100 \mu \mathrm{g})$ were separated by sodium dodecyl sulfate-polyacrylamide gel electrophoresis, transferred to nitrocellulose and probed with antibodies against AOX or coxll. was accompanied by a dramatic increase in the level of AOX protein detected in isolated leaf mitochondria by western analysis (Fig. 1B). Together, these results establish a dramatic induction of AOX in tobacco leaf in response to the shift to cold.

Previously, we described transgenic tobacco lines with suppressed levels of AOX protein (RI29, RI9, Amirsadeghi et al. 2006) or elevated levels of AOX protein (B7, B8, Vanlerberghe et al. 1998). Although WT plants contained large amounts of AOX protein at $48 \mathrm{~h}$ after transfer to cold, we did not detect any AOX protein at $48 \mathrm{~h}$ in RI29 and only low amounts in RI9, indicating an effective suppression of AOX (particularly in RI29), despite the strongly inducing conditions for Aox1a expression (Fig. 1B). As expected, B7 and B8 leaf mitochondria contained much higher levels of AOX protein than WT at both $28^{\circ} \mathrm{C} / 22^{\circ} \mathrm{C}$ and after transfer to $12^{\circ} \mathrm{C} / 5^{\circ} \mathrm{C}$, because of the strong constitutive expression of Aox1a in these plant lines (Fig. 1B).

\section{AOX level impacts cold-induced accumulation of leaf monosaccharides}

In Arabidopsis, cold is known to result in the accumulation of monosaccharides (Cook et al. 2004, Maruyama et al. 2009, Urano et al. 2010). We also saw a dramatic accumulation of leaf Glu (Fig. 2A) and Fru (Fig. 2B) in tobacco within $24 \mathrm{~h}$ of transfer to cold and some further accumulation occurred by $48 \mathrm{~h}$ and again by 72h. Interestingly, plants overexpressing AOX accumulated more Glu and Fru than WT, while plants with suppressed AOX expression accumulated less of these hexoses than WT. This pattern across plant lines was already evident at $24 \mathrm{~h}$ and was exaggerated further by 48 and 72 h. At 72 h, both overexpressing lines contained significantly higher levels of Glu and Fru than the WT and both suppressed lines contained significantly less of these monosaccharides than WT (Fig. 2A, B).

At $28^{\circ} \mathrm{C} / 22^{\circ} \mathrm{C}$, all plant lines displayed similar levels of leaf sucrose (Fig. 2C). Unlike the case with Glu and Fru, transfer to cold had little impact on overall sucrose level. However, after transfer the pattern of sucrose level across plant lines was similar to that seen with the hexoses, particularly by $72 \mathrm{~h}$. That is, lines overexpressing AOX tended to have slightly higher sucrose levels than WT and lines with suppressed AOX expression tended to have slightly less sucrose than WT. However, sucrose levels tended to be more variable and hence, in most cases, the differences were not statistically significant.

At $28^{\circ} \mathrm{C} / 22^{\circ} \mathrm{C}$, all plant lines displayed similar levels of leaf starch and transfer to cold resulted in an almost twofold increase in this level within 24 h (Fig. 2D). Some 

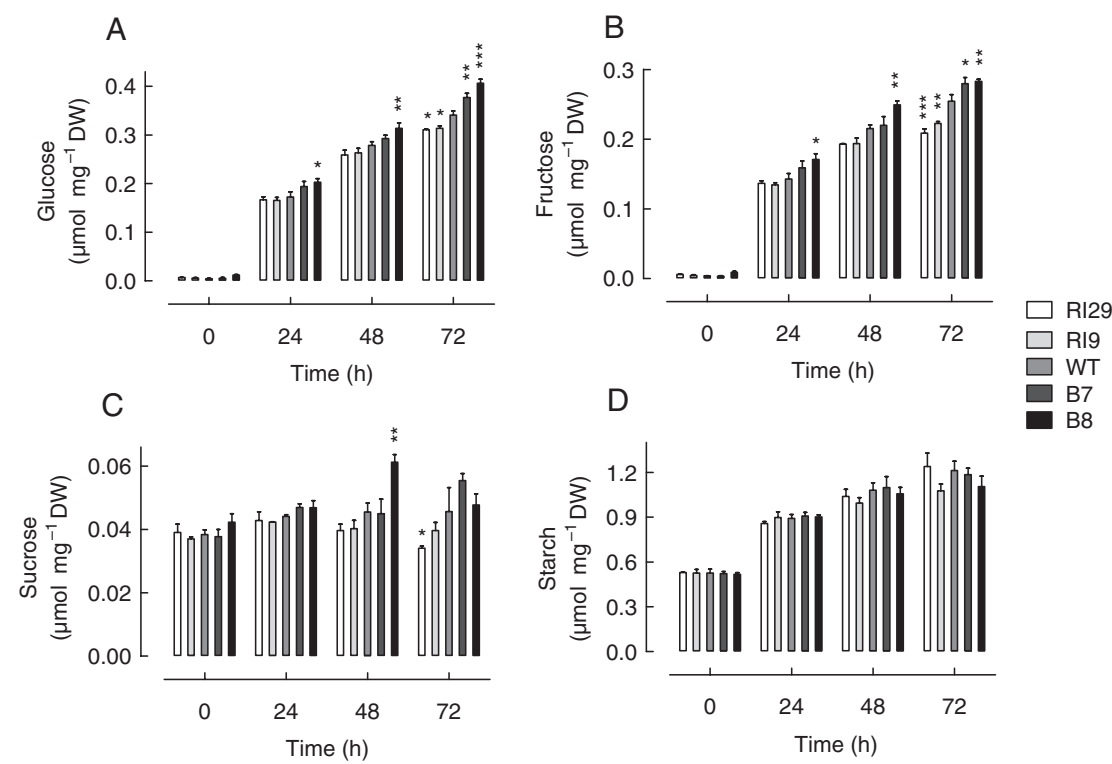

Fig. 2. Levels of Glu (A), Fru (B), sucrose (C) and starch (D) in the leaf of WT and transgenic tobacco at different times after transfer of plants from a growth temperature of $28^{\circ} \mathrm{C} / 22^{\circ} \mathrm{C}$ (light/dark) to a growth temperature of $12^{\circ} \mathrm{C} / 5^{\circ} \mathrm{C}$. The plant lines are ordered from left to right on the graph according to their relative level of AOX protein after transfer to cold (i.e. from lowest levels on the left to highest levels on the right). Data are the mean \pm SE of three independent experiments. Data were analyzed by two-way ANovA followed by a Bonferroni post-test to compare (within each time point) the WT line to each transgenic line. Number of asterisks above the bar indicates the level of significant difference: ${ }^{*} P<0.05$; ${ }^{* *} P<0.01$; *** $P<0.001$. Bars without an asterisk are not significantly different from the WT.

further increase in starch level was seen at $48 \mathrm{~h}$ and again at $72 \mathrm{~h}$, but no differences were seen across plant lines.

\section{AOX level impacts the level of leaf oxidative damage}

Lipid peroxidation, estimated as MDA level, is widely regarded as one of the most reliable indicators of the level of oxidative damage in plant tissues (Møller et al. 2007, Shulaev and Oliver 2006). At $28^{\circ} \mathrm{C} / 22^{\circ} \mathrm{C}$, plants overexpressing AOX had significantly lower levels of leaf MDA than WT, while plants with suppressed AOX expression displayed significantly higher levels of MDA than WT (Fig. 3A).

A more complex response of the plant lines was seen after transfer to cold. In RI29 (the line with the strongest suppression of AOX after transfer to cold; Fig. 1B), there was a significant decline in MDA at 24, 48 and $72 \mathrm{~h}$ after transfer to cold (Fig. 3B). None of the other lines responded in this fashion. In the WT and RI9 (lines with moderate to low AOX levels after transfer), there was a significant increase in MDA at $24 \mathrm{~h}$ (compared with $0 \mathrm{~h}$ ), while in B7 and B8 (lines with high levels of AOX before and after transfer) there was no significant change in MDA level after transfer (Fig. 3B). Fig. 3C shows the absolute change in MDA level for each line over the initial $24 \mathrm{~h}$ period after transfer to cold and illustrates that the change seen in RI29 (an overall decrease in
MDA) was significantly different than that seen in all the other lines, all of which saw an increase in MDA.

\section{AOX level impacts cold induction of components of the leaf ROS-scavenging network}

Given the dynamic changes in the MDA level of WT, RI9 and RI29 plants after transfer to cold (Fig. 3B, C), we also evaluated the response of components of the ROS-scavenging network. At $28^{\circ} \mathrm{C} / 22^{\circ} \mathrm{C}$, the transcript level of four key ROS-scavenging enzymes (CuZnSOD, FeSOD, GPX and APx) did not differ significantly between the WT and lines with suppressed AOX (Fig. 4). At $24 \mathrm{~h}$ after transfer to cold, the transcript level of CuZnSOD, GPx and APx had increased dramatically in WT, while that of FeSOD remained unchanged. Levels of CuZnSOD and GPx moderated somewhat by 48 and $72 \mathrm{~h}$, but were still above the levels seen at $28^{\circ} \mathrm{C} / 22^{\circ} \mathrm{C}$. Levels of APx continued to rise moderately throughout the $72 \mathrm{~h}$ period, while FeSOD levels continued to remain unchanged.

The transcript patterns seen in RI9 were almost identical to those in WT, except that RI9 did tend to have slightly higher levels (although not statistically significant) of CuZnSOD and FeSOD than WT, particularly by $72 \mathrm{~h}$ (Fig. 4). However, RI29 showed a stronger induction of all transcripts than WT. At 24 and $48 \mathrm{~h}$ after transfer, RI29 displayed significantly higher levels than 

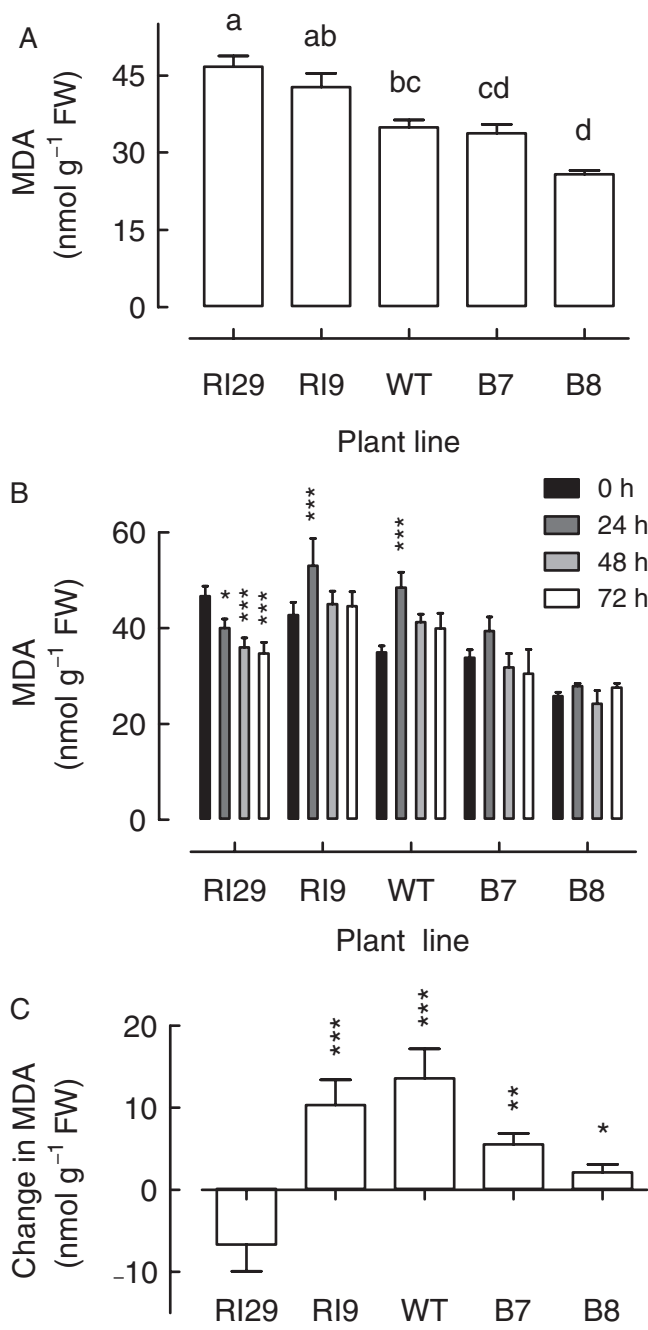

Plant line

Fig. 3. Levels of lipid peroxidation (measured as MDA equivalents) in the leaf of WT and transgenic tobacco. (A) Leaf MDA level in plants grown at $28^{\circ} \mathrm{C} / 22^{\circ} \mathrm{C}$ (light/dark). Data are the mean \pm SE of three independent experiments. Data were analyzed by one-way ANOva followed by a Bonferroni multiple comparison test. Bars not sharing a common letter $(a, b, c$ or $d)$ are significantly different from one another (i.e. $P<$ $0.05)$. (B) Leaf MDA level of WT and transgenic tobacco at different times after transfer of plants from a growth temperature of $28^{\circ} \mathrm{C} / 22^{\circ} \mathrm{C}$ (light/dark) to a growth temperature of $12^{\circ} \mathrm{C} / 5^{\circ} \mathrm{C}$. Data are the mean \pm SE of three independent experiments. Data were analyzed by two-way ANova followed by a Bonferroni post-test to compare (within each line) the level of MDA at $\mathrm{O}$ h to each other time point. Number of asterisks above the bar indicates the level of significant difference: $* P<0.05$; ${ }^{* *} P<0.01$; ${ }^{* * *} P<0.001$. Bars without an asterisk are not significantly different from time $0 \mathrm{~h}$. (C) The change in leaf MDA level in WT and transgenic tobacco over the first $24 \mathrm{~h}$ following transfer of plants from a growth temperature of $28^{\circ} \mathrm{C} / 22^{\circ} \mathrm{C}$ (light/dark) to a growth temperature of $12^{\circ} \mathrm{C} / 5^{\circ} \mathrm{C}$. These data (mean $\pm \mathrm{sE}$ ) are derived from the same data presented in (B). Data were analyzed by one-way Anova followed by a Dunnett's multiple comparison test, comparing each plant line with RI29. Number of asterisks above the bar indicates the level of significant difference: ${ }^{*} P<0.05 ;{ }^{* *} P<0.01 ;{ }^{* * *} P<0.001$
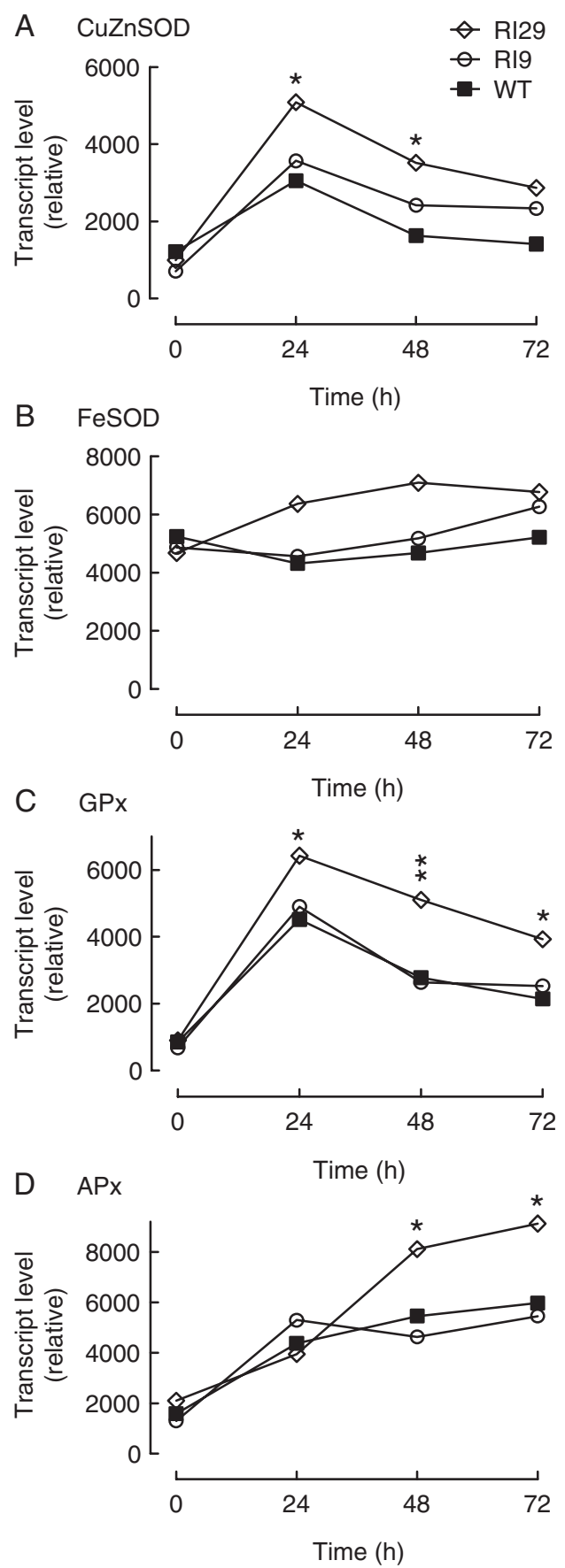

Fig. 4. Relative transcript (mRNA) level of genes encoding the ROSscavenging enzymes CuZnSOD (A), FeSOD (B), GPx (C) and APx (D) in leaves of WT (solid squares), RI9 (open circles) and RI29 (open diamonds) tobacco at different times after transfer of plants from a growth temperature of $28^{\circ} \mathrm{C} / 22^{\circ} \mathrm{C}$ (light/dark) to a growth temperature of $12^{\circ} \mathrm{C} / 5^{\circ} \mathrm{C}$. Data are the mean of two to three independent experiments. Data were analyzed by two-way Anova followed by a Bonferroni post-test to compare (within each time point) the WT line with each transgenic line. Number of asterisks above the symbol indicates the level of significant difference: ${ }^{*} P<0.05$; ${ }^{* *} P<0.01$; ${ }^{* * *} P<0.001$. Symbols without an asterisk are not significantly different from the WT. 


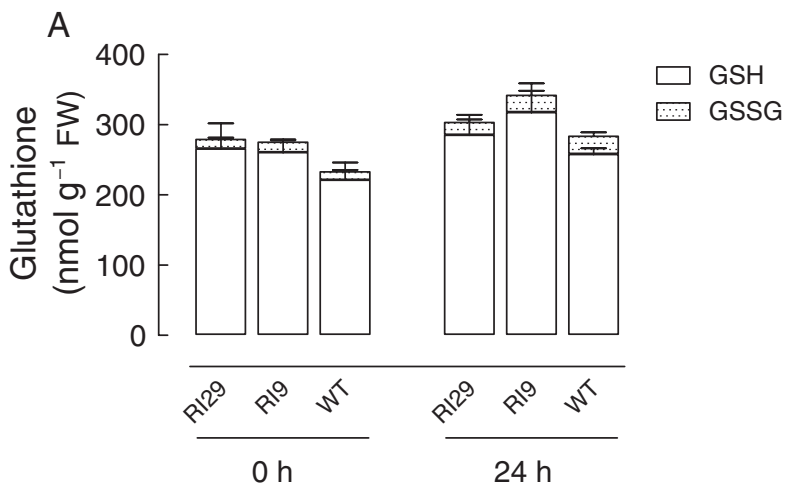

B

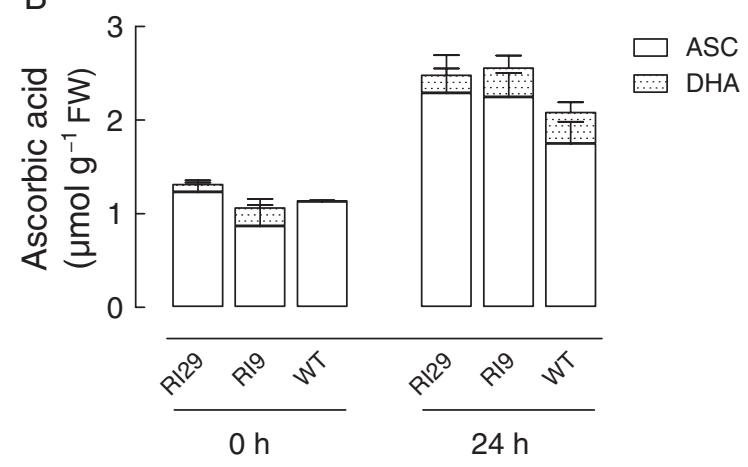

Fig. 5. Levels of oxidized glutathione (GSSG) and reduced glutathione (GSH) (A) and oxidized ascorbic acid (DHA) and reduced ascorbic acid (ASC) (B) in the leaf of WT and transgenic tobacco before transfer $(0 \mathrm{~h})$ and at $24 \mathrm{~h}$ after transfer from a growth temperature of $28^{\circ} \mathrm{C} / 22^{\circ} \mathrm{C}$ (light/dark) to a growth temperature of $12^{\circ} \mathrm{C} / 5^{\circ} \mathrm{C}$. Data are the mean \pm SE of three to four independent experiments. For each metabolite, data were analyzed by two-way Anova followed by a Bonferroni post-test to compare (within each time point) the metabolite level in the WT with the level in each transgenic line (RI9, RI29). These analyses did not identify any significant differences at the $P<0.05$ level.

WT of CuZnSOD and GPx. It also displayed significantly higher levels of APx by 48 and $72 \mathrm{~h}$. Also, while Wt plants showed no change in FeSOD transcript, the level of this transcript did tend to increase in RI29.

We also measured the levels of oxidized and reduced glutathione (Fig. 5A) as well as oxidized and reduced ascorbic acid (Fig. 5B) in order to evaluate the redox status of these important low-molecular weight antioxidants. Absolute concentrations of the different metabolites were similar to those seen previously in tobacco (Ding et al. 2009, Yabuta et al. 2002). Before and after transfer to cold, the level of all these metabolites were similar (i.e. not significantly different) in RI29 and RI9 compared with WT. Transfer to cold did lead to a modest oxidation of both the glutathione and ascorbic acid pools (as well as to a large increase in total ascorbic acid), but no significant differences were seen between the WT and suppressed lines.

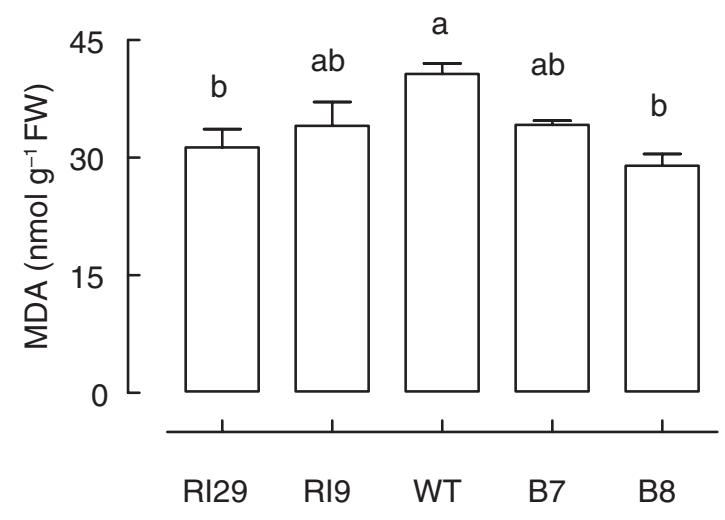

Plant line

Fig. 6. Levels of lipid peroxidation (measured as MDA equivalents) in the leaf of WT and transgenic tobacco grown at high PPFD $(700 \mu \mathrm{mol}$ $\mathrm{m}^{-2} \mathrm{~s}^{-1}$ ). Data are the mean $\pm \mathrm{SE}$ of three independent experiments. Data were analyzed by one-way Anova followed by a Newman-Keuls multiple comparison test. Bars not sharing a common letter (a or b) are significantly different from one another (i.e. $P<0.05$ ).

\section{The impact of AOX level on leaf oxidative damage is highly dependent upon growth conditions}

Levels of lipid peroxidation at $28^{\circ} \mathrm{C} / 22^{\circ} \mathrm{C}$ were also analyzed in plants grown at higher PPFD $(700 \mu \mathrm{mol}$ $\mathrm{m}^{-2} \mathrm{~s}^{-1}$ rather than the usual $120 \mu \mathrm{mol} \mathrm{m} \mathrm{m}^{-2} \mathrm{~s}^{-1}$ ). At high PPFD, RI29 and RI9 no longer displayed the higher levels of MDA (compared with WT) that was evident at the lower PPFD (compare Fig. 6 with Fig. 3A). This indicates that PPFD is another growth parameter (besides temperature) that alters the relative levels of lipid peroxidation between the plant lines expressing different levels of AOX.

\section{Discussion}

Respiration typically couples the breakdown of carbohydrate with the synthesis of ATP by oxidative phosphorylation. However, as AOX respiration is not directly associated with such energy conservation, it provides flexibility to the otherwise tight coupling among carbon metabolism, electron transport and ATP turnover (Finnegan et al. 2004). We found that transfer of warm-grown tobacco plants to cold resulted in a large induction of leaf AOX (Fig. 1), similar to previous results seen with tobacco suspension cells (Vanlerberghe and McIntosh 1992). To investigate the physiological role of this large induction of leaf AOX, we compared the response to cold of WT plants with that of transgenic plants with modified AOX levels.

One physiological role of AOX could be to burn excess carbohydrate, without this process being constrained 
by rates of ATP turnover (Gandin et al. 2009, Lambers 1982, Noguchi and Terashima 2006, Simons and Lambers 1999). We found that WT tobacco plants showed a dramatic increase of Glu and Fru upon transfer from a growth temperature of $28^{\circ} \mathrm{C} / 22^{\circ} \mathrm{C}$ (light/dark) to $12^{\circ} \mathrm{C} / 5^{\circ} \mathrm{C}$ (Fig. 2A, B). This provided an interesting experimental system to test the impact of AOX on carbohydrate level. If AOX acts to burn excess carbohydrate, we hypothesized that transgenic plants lacking AOX might accumulate greater amounts of carbohydrate than the WT after transfer to cold, while overexpression of AOX might compromise the rapid carbohydrate accumulation. However, we found just the opposite. Plants lacking AOX actually accumulated significantly less carbohydrate than the WT, while plants overexpressing AOX accumulated significantly more (Fig. 2A, B).

The above results are not consistent with the hypothesis that AOX acts to burn excess carbohydrate but rather suggests a role for AOX in aiding sugar accumulation in response to the cold stress. As sugar accumulation in response to cold may be dependent upon active photosynthesis, one possibility is that AOX aids sugar accumulation by having a positive impact on photosynthetic metabolism under cold. Some work does suggest that lack of AOX can impede photosynthesis under various conditions (Giraud et al. 2008, Noguchi and Yoshida 2008, Raghavendra and Padmasree 2003, Strodtkötter et al. 2009, Yoshida et al. 2007) and a recent study with field-grown grasses found a positive correlation between electron flow to AOX and the previous days PPFD (Searle et al. 2010). Also, it is previously shown that tobacco plants lacking AOX show increased expression of plastid terminal oxidase, perhaps an indication of perturbed photosynthetic metabolism (Amirsadeghi et al. 2006). Our future studies will investigate the potential role of AOX to facilitate photosynthesis under cold. It should be noted that our results differ from those reported for Arabidopsis, where WT plants accumulated large amounts of starch and displayed a dramatic increase in $C / N$ ratio upon transfer to cold $\left(4^{\circ} \mathrm{C}\right)$, and where these effects were enhanced in a knock-out mutant lacking Aox1a (Watanabe et al. 2008).

Another central hypothesis for the physiological role of $\mathrm{AOX}$ is that it may act to dampen ROS generation by the mitochondrial ETC (Purvis and Shewfelt 1993). This has been directly demonstrated in transgenic tobacco suspension cell cultures, where a lack of AOX was accompanied by increased ROS emanating specifically from the mitochondrion (Maxwell et al. 1999). Consistent with this hypothesis, we found that, at least when plants were grown at low PPFD $(120 \mu \mathrm{mol}$ $\left.\mathrm{m}^{-2} \mathrm{~s}^{-1}\right)$ and warm temperature $\left(28^{\circ} \mathrm{C} / 22^{\circ} \mathrm{C}\right)$, transgenic plants lacking AOX displayed higher levels of oxidative damage (MDA) than WT, while plants overexpressing AOX displayed less oxidative damage than WT (Fig. 3A). These results are suggestive of higher rates of ROS generation in plants lacking AOX and lower rates of ROS generation in plants overexpressing AOX, similar to the results seen in suspension cells. It is also consistent with the studies of isolated mitochondria showing that AOX respiration lowers rates of mitochondrial ROS generation (Popov et al. 1997).

We initially hypothesized that the higher levels of oxidative damage seen at $28^{\circ} \mathrm{C} / 22^{\circ} \mathrm{C}$ in AOX-suppressed lines compared with WT would become further exaggerated after transfer to cold as the difference in AOX level between the WT and suppressed lines becomes greatly exaggerated after the cold stress. Also, the role of AOX to dampen ROS generation is often hypothesized to be of particular importance under stress conditions as stress may result in imbalances in carbon and energy metabolism, while the flexibility provided by the non-energy conserving nature of AOX respiration could act to alleviate such imbalances (Finnegan et al. 2004, Simons and Lambers 1999). However, we found that the differences in MDA level among RI29, RI9 and WT were not exaggerated but rather were largely nullified by the transfer to cold, primarily because of a decrease (rather than increase) of MDA in RI29 and similar increases of MDA in RI9 and WT (Fig. 3B, C).

It is widely held that cellular ROS level (and by extension, the level of oxidative damage such as MDA level) is determined not solely by rates of ROS generation but rather by the balance between rates of ROS generation and rates of ROS scavenging (Apel and Hirt 2004, Foyer and Noctor 2009, Møller 2001). Examining the transcript level of key ROS-scavenging enzymes, we found no differences between the WT and AOX-suppressed lines when grown at $28^{\circ} \mathrm{C} / 22^{\circ} \mathrm{C}$ (Fig. 4). However, upon transfer to cold, the level of these transcripts was enhanced much more in RI29 than either the WT or RI9 and only moderately more (not significant) in RI9 than WT. This corresponds well with the trends seen in MDA level, in which MDA level actually dropped significantly in RI29 after transfer to cold while rising in both RI9 and WT (Fig. 3C). That RI29 was able to effectively lower levels of oxidative damage is in keeping with the data showing that ascorbic acid and glutathione pools remained highly reduced after the shift to cold (Fig. 5). Hence, these pools would remain effective in supporting an increased rate of ROS scavenging.

On the basis of the above results, we suggest that the growth at $28^{\circ} \mathrm{C} / 22^{\circ} \mathrm{C}$ represents a 'low-stress' or 'non-stress' condition in which the lack of AOX in RI9 and RI29 does moderately increase ROS generation (hence leading to some increase in MDA relative to 
WT; Fig. 3A), but not to a presumed threshold condition necessary to signal upregulation of the ROS-scavenging network in comparison to WT. However, the shift to cold appears to represent a 'stress' condition for respiratory metabolism, one in which the WT responds by inducing large amounts of the stress-responsive Aox 1 a transcript and AOX protein (Fig. 1). Under these conditions, the strong suppression of AOX in RI29 is potentially much more detrimental but instead results in a threshold condition being achieved that signals upregulation of the ROS-scavenging network. Hence, RI29 plants under these growth conditions display a stronger than WT upregulation of the ROS-scavenging network (and perhaps other acclimations) that actually overcompensate for the lack of AOX, resulting paradoxically in lower levels of oxidative damage (Fig. 3C). RI9 plants, however, do not display as strong a suppression of AOX as RI29 following transfer to cold (Fig. 1B). As a result, these plants do not appear to reach the threshold condition needed for a stronger than WT induction of the ROS-scavenging network (Fig. 4) and hence display oxidative damage similar to or slightly greater than the WT after transfer to cold (Fig. 3B). Fig. 7 summarizes the aspects of this model.

The model (Fig. 7) would appear to apply to other observations we have made as well. Previously, we showed that RI29 and RI9 plants (grown at $28^{\circ} \mathrm{C} / 22^{\circ} \mathrm{C}$ ) maintained higher transcript levels than WT of manganese superoxide dismutase, CuZnSOD, APx, catalase and FeSOD (Amirsadeghi et al. 2006), while it was clear in the present study that transcript levels of ROSscavenging enzymes were not greater in RI29 and RI9 than the WT at $28^{\circ} \mathrm{C} / 22^{\circ} \mathrm{C}$ (Fig. 4). However, a major difference in the growth conditions between the two studies is that plants analyzed by Amirsadeghi et al. (2006) were grown at a much higher PPFD $(400 \mu \mathrm{mol}$ $\left.\mathrm{m}^{-2} \mathrm{~s}^{-1}\right)$ than in the current study $\left(120 \mu \mathrm{mol} \mathrm{m}^{-2} \mathrm{~s}^{-1}\right)$. We found that this difference in the relative induction level between lines of the ROS-scavenging network because of PPFD corresponds with differences in the relative level of oxidative damage between lines. At low PPFD, WT, RI9 and RI29 have a similar status of their ROS-scavenging network (at least in terms of transcript levels of these important ROS-scavengers; Fig. 4) and the suppression of AOX (in RI9 and RI29) under these conditions clearly results in higher levels of MDA than found in the WT (Fig. 3A). On the other hand, at higher PPFD the ROS-scavenging network is induced in RI9 and RI29 in comparison to WT (Amirsadeghi et al. 2006) and we find that this results in a lower level of MDA in RI9 and RI29 than found in the WT (Fig. 6). These results suggest that, like the transfer to cold, growth at higher PPFD can be considered a 'stress' condition for
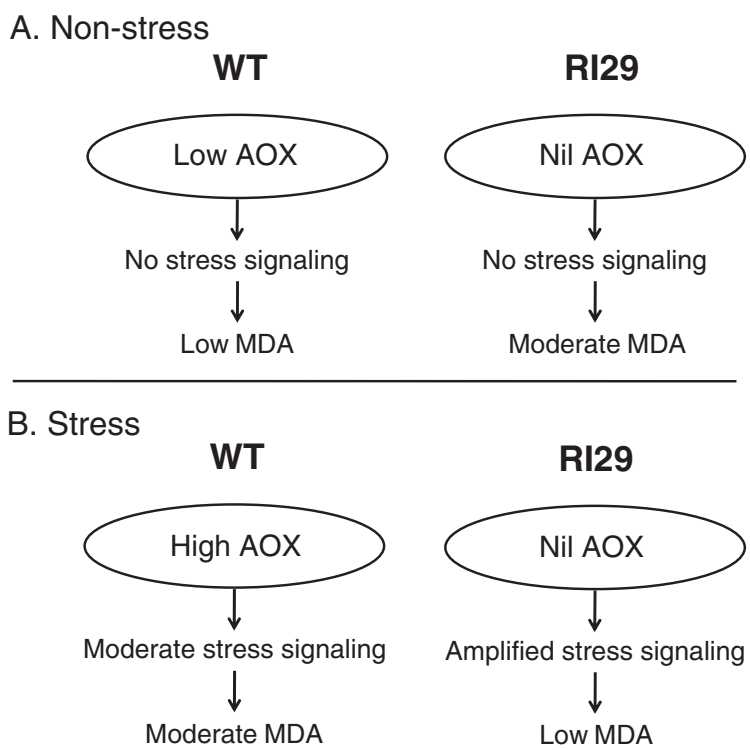

Fig. 7. A working model for the impact of AOX level on ROS metabolism, mitochondrial signaling and oxidative damage in tobacco leaf. (A) Under non-stress conditions, WT tobacco maintains low levels of mitochondrial AOX but the ROS-scavenging system is nonetheless sufficient to maintain relatively low levels of MDA. However, in strongly AOX-suppressed plants (RI29) there is some increase in the rate of ROS generation (presumably from the mitochondrial ETC) compared with WT, but the new rate is not sufficient under these non-stress conditions to signal upregulation of the ROS-scavenging network, resulting in elevated MDA compared with WT. (B) Transfer to cold temperature (or high PPFD) represents a stress condition for respiratory metabolism and WT plants respond by inducing large amounts of AOX. This is important to dampening ROS generation, which when combined with a moderate increase in the ROS-scavenging network is sufficient to moderate increases in MDA. In AOX-suppressed plants, the inability to induce $A O X$ results in much higher rates of ROS generation such that a signaling threshold is achieved whereby the ROS-scavenging network (and perhaps other systems) is amplified (in comparison to WT) and overcompensates for the new rate of ROS generation, resulting paradoxically in a lowering of MDA in comparison to the non-stress condition. See text for further details.

respiratory metabolism that, in WT plants, is alleviated by the enhancement of AOX level, but which in plants with suppressed AOX results in an upregulation of the ROS-scavenging network. In fact, studies have shown increasing levels of AOX with increasing PPFD (Dinakar et al. 2010, Yoshida et al. 2007) and we have observed this to be the case in tobacco as well (Cheung and Vanlerberghe, unpublished data).

Our results may provide explanation for some of the disparate results seen in reverse genetic experiments with Arabidopsis. For example, Fiorani et al. (2005) found that, at low temperature $\left(12^{\circ} \mathrm{C}\right), \mathrm{AOX}$-suppressed lines had higher MDA than WT and there was no enhanced activation of the ROS-scavenging network compared with WT. Contrary to this, Watanabe et al. (2008) 
reported that, at $4{ }^{\circ} \mathrm{C}$, Arabidopsis lacking AOX had lower MDA than WT and showed enhanced activation of the ROS-scavenging network compared with WT. These disparate results could be because of the differences in the low temperature treatments between the two studies or could be because of differences between the AOX lines used [i.e. the AOX-suppressed line used by Fiorani et al. (2005) still maintained significant levels of AOX (approximately $27 \%$ of WT), while those used by Watanabe et al. (2008) had no detectable AOX]. Similarly, in the current study, two tobacco lines with differing levels of AOX suppression (RI9, RI29) responded differentially to the same low temperature treatment (Fig. 3C). This would favor the conclusion that in tobacco (and likely Arabidopsis as well), a strong suppression of AOX under stress conditions can induce defenses that 'overcompensate' for the lack of AOX, at least in terms of ROS scavenging. Interestingly, such overcompensation effects have also been seen in other studies involving manipulation of components of the ROS network (Rizhsky et al. 2002), suggesting that this might be a common phenomenon.

The observation that manipulation of the composition of the mitochondrial ETC (such as done here with AOX level) alters plant acclimatory responses to stress (in this case the response of the cellular ROS-scavenging system in RI29 to a transfer to cold) favors the concept that the mitochondrion is able to signal its status to other parts of the cell and hence initiate cellular responses to stress (Kuzmin et al. 2004, Noctor et al. 2004, 2007). ROS themselves are often implicated as a signaling molecule within such cascade(s) (Apel and Hirt 2004, Foyer and Noctor 2009, Suzuki and Mittler 2006) and as AOX likely influences the rate of ROS generation by the mitochondrial ETC, it would in fact be uniquely positioned to impact (and perhaps even modulate) such a signal path(s) (Pasqualini et al. 2007, Van Aken et al. 2009, Vanlerberghe et al. 2009).

Reverse genetic experiments in both Arabidopsis (Fiorani et al. 2005, Watanabe et al. 2008) and tobacco (this study) indicate that Aox1a has a role in both carbon metabolism and the ROS network and that growth parameters such as temperature are an important factor impacting its role. This is in keeping with the observation that the expression of Aox1a is particularly responsive to growth conditions (at least in those plant species studied), while other AOX gene family members appear to be important for particular developmental stages (Clifton et al. 2006, Chai et al. 2010, Saisho et al. 2001, Wang and Vanlerberghe, unpublished data).

A large accumulation of total ascorbic acid (approximately twofold) occurred within the first $24 \mathrm{~h}$ after transfer of tobacco to the cold, suggestive of rapid ascorbic acid biosynthesis (Fig. 5B). The oxidation of
L-galactone-1,4-lactone to ascorbic acid is coupled to the reduction of cytochrome $c$ in a reaction catalyzed by the inner mitochondrial membrane enzyme L-galactone1,4-lactone dehydrogenase (Bartoli et al. 2000, Millar et al. 2003). Using transgenic Arabidopsis plants with altered levels of AOX, it was shown that AOX promoted rates of ascorbic acid synthesis, presumably as a result of AOX increasing the availability of oxidized cytochrome C (Bartoli et al. 2006). However, we found no differences between WT and AOX-suppressed tobacco lines in the leaf total pool size of ascorbic acid, either before or $24 \mathrm{~h}$ after transfer to cold. This indicates that, at least in tobacco and under our growth conditions, AOX was dispensable to maintaining normal levels of ascorbic acid, even during a period of rapid increase in ascorbic acid pool size following transfer to cold.

In conclusion, tobacco shows a large induction of leaf AOX after transfer to lower growth temperature. On the basis of the results with transgenic plants, this large induction of AOX appears to aid cold-induced monosaccharide accumulation as well as acting to stabilize ROS generation and hence the need for the cellular ROS-scavenging network. More broadly, the results also support the idea that the mitochondrion is a target of low temperature in tobacco and that mitochondrial condition can influence (perhaps via a ROS-based signaling pathway originating from the mitochondrion) the induction state of the cellular ROS-scavenging network.

Acknowledgement - The authors acknowledge financial support for this work from the Natural Sciences and Engineering Research Council of Canada to G. C. V.

\section{References}

Amirsadeghi S, Robson CA, McDonald AE, Vanlerberghe GC (2006) Changes in plant mitochondrial electron transport alter cellular levels of reactive oxygen species and susceptibility to cell death signaling molecules. Plant Cell Physiol 47: 1509-1519

Armstrong AF, Badger MR, Day DA, Barthet MM, Smith PMC, Millar AH, Whelan J, Atkin OK (2008) Dynamic changes in the mitochondrial electron transport chain underpinning cold acclimation of leaf respiration. Plant Cell Environ 31: 1156-1169

Apel K, Hirt H (2004) Reactive oxygen species: metabolism, oxidative stress, and signal transduction. Ann Rev Plant Biol 55: 373-399

Bartoli CG, Pastori GM, Foyer CH (2000) Ascorbate biosynthesis in mitochondria is linked to the electron transport chain between complexes III and IV. Plant Physiol 123: 335-343 
Bartoli CG, Yu J, Gómez F, Fernández L, Mclntosh L, Foyer $\mathrm{CH}$ (2006) Inter-relationships between light and respiration in the control of ascorbic acid synthesis and accumulation in Arabidopsis thaliana leaves. J Exp Bot 57: $1621-1631$

Campbell C, Atkinson L, Zaragoza-Castells J, Lundmark M, Atkin O, Hurry V (2007) Acclimation of photosynthesis and respiration is asynchronous in response to changes in temperature regardless of plant functional group. New Phytol 176: 375-389

Chai T-T, Simmonds D, Day DA, Colmer TD, Finnegan PM (2010) Photosynthetic performance and fertility are repressed in GmAOX2b antisense soybean. Plant Physiol 152: 1638-1649

Clifton R, Lister R, Parker KL, Sappl PG, Elhafez D, Millar AH, Day DA, Whelan J (2005) Stress-induced co-expression of alternative respiratory chain components in Arabidopsis thaliana. Plant Mol Biol 58: 193-212

Clifton R, Millar AH, Whelan J (2006) Alternative oxidases in Arabidopsis: a comparative analysis of differential expression in the gene family provides new insights into function of non-phosphorylating bypasses. Biochim Biophys Acta 1757: 730-741

Cook D, Fowler S, Fiehn O, Thomashow MF (2004) A prominent role for the CBF cold response pathway in configuring the low-temperature metabolome of Arabidopsis. PNAS 101: 15243-15248

Dinakar C, Raghavendra AS, Padmasree K (2010) Importance of AOX pathway in optimizing photosynthesis under high light stress: role of pyruvate and malate in activating AOX. Physiol Plant 139: 13-26

Ding S, Lu Q, Zhang Y, Yang Z, Wen X, Zhang L, Lu C (2009) Enhanced sensitivity to oxidative stress in transgenic tobacco plants with decreased glutathione reductase activity leads to a decrease in ascorbate pool and ascorbate redox state. Plant Mol Biol 69: 577-592

Finnegan PM, Soole KL, Umbach AL (2004) Alternative mitochondrial electron transport proteins in plants. In: Day DA, Millar AH, Whelan J (eds) Plant Mitochondria: From Genome to Function. Kluwer Academic Publishers, Dordrecht, pp 163-230

Fiorani F, Umbach AL, Siedow JN (2005) The alternative oxidase of plant mitochondria is involved in the acclimation of shoot growth at low temperature. A study of Arabidopsis AOX1a transgenic plants. Plant Physiol 139: 1795-1805

Foyer CH, Noctor G (2009) Redox regulation in photosynthetic organisms: signaling, acclimation, and practical implications. Antioxidant Redox Signal 11: $861-905$

Gandin A, Lapointe L, Dizengremel P (2009) The alternative respiratory pathway allows sink to cope with changes in carbon availability in the sink-limited plant Erythronium americanum. J Exp Bot 60: 4235-4248
Giraud E, Ho LHM, Clifton R, Carroll A, Estavillo G, Tan Y-F, Howell KA, Ivanova A, Pogson BJ, Millar AH, Whelan J (2008) The absence of ALTERNATIVE OXIDASE1a in Arabidopsis results in acute sensitivity to combined light and drought stress. Plant Physiol 147: 595-610

Gonzàlez-Meler MA, Ribas-Carbo M, Giles L, Siedow JN (1999) The effect of growth and measurement temperature on the activity of the alternative respiratory pathway. Plant Physiol 120: 765-772

Guy RD, Vanlerberghe GC (2005) Partitioning of respiratory electrons in the dark in leaves of transgenic tobacco with modified levels of alternative oxidase. Physiol Plant 125: 171-180

Hara M, Terashima S, Fukaya T, Kuboi T (2003) Enhancement of cold tolerance and inhibition of lipid peroxidation by citrus dehydrin in transgenic tobacco. Planta 217: 290-298

Hoagland DR, Arnon DI (1950) The water-culture method for growing plants without soil. Calif Agric Exp Sta Circ 347

Ito Y, Saisho D, Nakazono M, Tsutsumi N, Hirai A (1997) Transcript levels of tandem-arranged alternative oxidase genes in rice are increased by low temperature. Gene 203: $121-129$

Jones MG (1981) Enzymic assay for starch and glycogen. Tech Carbohyd Metab 303: 1-13

Kuzmin EV, Karpova OV, Elthon TE, Newton KJ (2004) Mitochondrial respiratory deficiencies signal up-regulation of genes for heat shock proteins. J Biol Chem 279: 20672-20677

Lambers H (1982) Cyanide-resistant respiration: a non-phosphorylating electron transport pathway acting as an energy overflow. Physiol Plant 55: 478-485

MacFarlane C, Hansen LD, Florez-Sarasa I, Ribas-Carbo M (2009) Plant mitochondria electron partitioning is independent of short-term temperature changes. Plant Cell Environ 32: 585-591

Maruyama K, Takeda M, Kidokoro S, Yamada K, Sakuma Y, Urano K, Fujita M, Yoshiwara K, Matsukura S, Morishita Y, Sasaki R, Suzuki H, Saito K, Shibata D, Shinozaki K, Yamaguchi-Shinozaki K (2009) Metabolic pathways involved in cold acclimation identified by integrated analysis of metabolites and transcripts regulated by DREB1A and DREB2A. Plant Physiol 150: 1972-1980

Maxwell DP, Wang Y, Mclntosh L (1999) The alternative oxidase lowers mitochondrial reactive oxygen production in plant cells. Proc Natl Acad Sci USA 96: $8271-8276$

Millar AH, Mittova V, Kiddle G, Heazlewood JL, Bartoli CTG, Theodoulou FL, Foyer CH (2003) Control of ascorbate synthesis by respiration and its implications for stress responses. Plant Physiol 133: 443-447 
Miller RE, Grant NM, Giles L, Ribas-Carbo M, Berry JA, Watling JR, Robinson SA (2011) In the heat of the night - alternative pathway respiration drives thermogenesis in Philodendron bipinnatifidum. New Phytol 189: 1013-1026. doi: 10.1111/j.1469-8137. 2010.03547.x

Mizuno N, Sugie A, Kobayashi F, Takumi S (2008) Mitochondrial alternative pathway is associated with development of freezing tolerance in common wheat. J Plant Physiol 165: 462-467

Møller IM (2001) Plant mitochondria and oxidative stress: electron transport, NADPH turnover, and metabolism of reactive oxygen species. Annu Rev Plant Physiol Plant Mol Biol 52: $561-591$

Møller IM, Jensen PE, Hansson A (2007) Oxidative modifications to cellular components in plants. Annu Rev Plant Biol 58: 459-481

Noctor G, Dutilleul C, De Paepe R, Foyer CH (2004) Use of mitochondrial electron transport mutants to evaluate the effects of redox state on photosynthesis, stress tolerance and the integration of carbon/nitrogen metabolism. J Exp Bot 55: 49-57

Noctor G, De Paepe R, Foyer CH (2007) Mitochondrial redox biology and homeostasis in plants. Trend Plant Sci 12: $125-134$

Noguchi K, Terashima I (2006) Responses of spinach leaf mitochondria to low $\mathrm{N}$ availability. Plant Cell Environ 29: 710-719

Noguchi K, Yoshida K (2008) Interaction between photosynthesis and respiration in illuminated leaves. Mitochondrion 8: 87-99

Pasqualini S, Paolocci F, Borgogni A, Morettini R, Ederli L (2007) The overexpression of an alternative oxidase gene triggers ozone sensitivity in tobacco plants. Plant Cell Environ 30: 1545-1556

Popov VN, Simonian RA, Skulachev VP, Starkov AA (1997) Inhibition of the alternative oxidase stimulates $\mathrm{H}_{2} \mathrm{O}_{2}$ production in plant mitochondria. FEBS Lett 415: $87-90$

Purvis AC, Shewfelt RL (1993) Does the alternative pathway ameliorate chilling injury in sensitive plant tissues? Physiol Plant 88: 712-718

Queval G, Noctor G (2007) A plate reader method for the measurement of NAD, NADP, glutathione and ascorbate in tissue extracts. Application to redox profiling during Arabidopsis rosette development. Anal Biochem 363: 58-69

Raghavendra AS, Padmasree K (2003) Beneficial interactions of mitochondrial metabolism with photosynthetic carbon assimilation. Trends Plant Sci 8: 546-553

Ribas-Carbo M, Aroca R, Gonzàlez-Meler MA, Irigoyen JJ, Sánchez-Díaz M (2000) The electron partitioning between the cytochrome and alternative respiratory pathways during chilling recovery in two cultivars of maize differing in chilling sensitivity. Plant Physiol 122: 199-204

Rizhsky L, Hallak-Herr E, Van Breusegem F, Rachmilevitch S, Barr JE, Rodermel S, Inze D, Mittler R (2002) Double antisense plants lacking ascorbate peroxidase and catalase are less sensitive to oxidative stress than single antisense plants lacking ascorbate peroxidase or catalase. Plant J 32: 329-342

Robson CA, Vanlerberghe GC (2002) Transgenic plant cells lacking mitochondrial alternative oxidase have increased susceptibility to mitochondria-dependent and -independent pathways of programmed cell death. Plant Physiol 129: 1908-1920

Saisho D, Nakazono M, Lee K-H, Tsutsumi N, Akita S, Hirai A (2001) The gene for alternative oxidase-2 (AOX2) from Arabidopsis thaliana consists of five exons unlike other $A O X$ genes and is transcribed at an early stage during germination. Genes Genet Syst 76:89-97 Searle SY, Thomas S, Griffin KL, Horton T, Kornfeld A, Yakir D, Hurry V, Turnbull MH (2010) Leaf respiration and alternative oxidase in field-grown alpine grasses respond to natural changes in temperature and light. New Phytol 189: 1027-1039

Shulaev V, Oliver DJ (2006) Metabolic and proteomic markers for oxidative stress. New tools for reactive oxygen species research. Plant Physiol 141: 367-372

Sieger SM, Kristensen BK, Robson CA, Amirsadeghi S, Eng EWY, Abdel-Mesih A, Møller IM, Vanlerberghe GC (2005) The role of alternative oxidase in modulating carbon use efficiency and growth during macronutrient stress in tobacco cells. J Exp Bot 56: 1499-1515

Simons BH, Lambers H (1999) The alternative oxidase: is it a respiratory pathway allowing a plant to cope with stress? In: Lerner HR (ed) Plant Responses to Environmental Stress: From Phytohormones to Gene Reorganization. Marcel Dekker Inc, New York, pp 265-286

Stitt M, Lilley RM, Gerhardt R, Heldt HW (1989) Metabolite levels in specific cells and subcellular compartments of plant leaves. Meth Enzymol 174: 518-552

Strodtkötter I, Padmasree K, Dinakar C, Speth B, Niazi PS, Wojtera J, Voss I, Do PT, Nunes-Nesi A, Fernie AR, Linke V, Raghavendra AS, Scheibe R (2009) Induction of the AOX1D isoform of alternative oxidase in A. thaliana T-DNA insertion lines lacking isoform AOX1A is insufficient to optimize photosynthesis when treated with antimycin A. Mol Plant 2: 284-297

Sugie A, Naydenov N, Mizuno N, Nakamura C, Takumi S (2006) Overexpression of wheat alternative oxidase gene Waox $1 \mathrm{a}$ alters respiration capacity and response to reactive oxygen species under low temperature in transgenic Arabidopsis. Genes Genet Syst 81: 349-354 
Suzuki N, Mittler R (2006) Reactive oxygen species and temperature stresses: a delicate balance between signaling and destruction. Physiol Plant 126: 45-51

Taylor NL, Day DA, Millar AH (2002) Environmental stress causes oxidative damage to plant mitochondria leading to inhibition of glycine decarboxylase. J Biol Chem 277: 42663-42668

Umbach AL, Lacey EP, Richter SJ (2009)

Temperature-sensitive alternative oxidase protein content and its relationship to floral reflectance in natural Plantago lanceolata populations. New Phytol 181: 662-671

Urano K, Kurihara Y, Seki M, Shinozaki K (2010) "Omics" analyses of regulatory networks in plant abiotic stress responses. Curr Opin Plant Biol 13: 132-138

Usadel B, Bläsing OE, Gibon Y, Poree F, Höhne M, Günter M, Trethewey R, Kamlage B, Poorter H, Stitt M (2008) Multilevel genomic analysis of the response of transcripts, enzyme activities and metabolites in Arabidopsis rosettes to a progressive decrease of temperature in the non-freezing range. Plant Cell Environ 31: 518-547

Van Aken O, Giraud E, Clifton R, Whelan J (2009) Alternative oxidase: a target and regulator of stress responses. Physiol Plant 137: 354-361

Vanessa DRF, Angela PT, Mariana CO, Pio C (2008) RNA isolation method for polysaccharide rich algae: agar producing Gracilaria tenuistipitata (Rhodophyta). J Appl Phycol 20: 9-12

Vanlerberghe GC, Mclntosh L (1992) Lower growth temperature increases alternative pathway capacity and alternative oxidase protein in tobacco. Plant Physiol 100: 115-119
Vanlerberghe GC, McIntosh L (1994) Mitochondrial electron transport regulation of nuclear gene expression: studies with the alternative oxidase gene of tobacco. Plant Physiol 105: 867-874

Vanlerberghe GC, Day DA, Wiskich JT, Vanlerberghe AE, Mclntosh L (1995) Alternative oxidase activity in tobacco leaf mitochondria: dependence on tricarboxylic acid cycle-mediated redox regulation and pyruvate activation. Plant Physiol 109: $353-$ 361

Vanlerberghe GC, Mclntosh L, Yip JYH (1998) Molecular localization of a redox-modulated process regulating plant mitochondrial electron transport. Plant Cell 10: 1551-1560

Vanlerberghe GC, Cvetkovska M, Wang J (2009) Is the maintenance of homeostatic mitochondrial signaling during stress a physiological role for alternative oxidase? Physiol Plant 137: 392-406

Watanabe CK, Hachiya T, Terashima I, Noguchi K (2008) The lack of alternative oxidase at low temperature leads to a disruption of the balance in carbon and nitrogen metabolism, and to an up-regulation of antioxidant defense systems in Arabidopsis thaliana leaves. Plant Cell Environ 31: 1190-1202

Yabuta Y, Motoki T, Yoshimura K, Takeda T, Ishikawa T, Shigeoka S (2002) Thylakoid membrane-bound ascorbate peroxidase is a limiting factor of antioxidative systems under photo-oxidative stress. Plant J 32: 915-925

Yoshida K, Terashima I, Noguchi K (2007) Up-regulation of mitochondrial alternative oxidase concomitant with chloroplast over-reduction by excess light. Plant Cell Physiol 48: 606-614

Edited by V. Hurry 